Audit of appropriate consideration of anti-craving medication following alcohol detoxification in a north east addictions service

Joseph Thorne* and Sophie Quarshie

Cumbria, Northumberland, Tyne And Wear NHS Foundation Trust *Corresponding author.

doi: 10.1192/bjo.2021.936

Aims. NICE guidelines recommend that all patients who undergo a successful alcohol detoxification programme should be considered for treatment with acamprosate or oral naltrexone. This audit studied the proportion of patients considered for acamprosate or naltrexone treatment in a North-East Addictions Service.

Primary aim

To explore whether naltrexone/acamprosate had been considered for each patient completing alcohol detoxification.

Secondary aims

what proportion of those offered agreed to be prescribed acamprosate/naltrexone

whether these patients were being adequately followed up in terms of prescription

Background. There is a significant evidence base for both naltrexone and acamprosate in the maintenance of abstinence in patients with alcohol addiction. NICE recommends the consideration of both medications for patients following successful alcohol detoxification from alcohol. The addictions service at Plummer Court in Newcastle upon Tyne has a comprehensive pathway for alcohol detoxification patients, which involves multiple reviews by keyworkers and medics. The attendance at these appointments is often poor, and it is often unclear whether these patients have been offered anti-craving medication.

Method. A list of patients referred for inpatient or outpatient alcohol detoxification between June to August $2018 \quad(n=23)$ was curated. The progress notes were reviewed for any evidence that there had been clinical consideration of acamprosate/ naltrexone. If evidence was found that the discussion had taken place, the notes were further scrutinised to assess if the client had accepted a prescription. The clinical documentation was further reviewed to see if follow-up for anti-craving medication was in place.

Result. There was evidence that anti-craving medication had been considered in $47 \%$ of patients during the treatment process

In all but one case, acamprosate was offered rather than naltrexone

In cases where medication was offered, it was accepted in all but one case

Anti-craving medication was universally well tolerated

There was considerable difficulty with assessing who was following up the prescription. On scrutiny of the notes, several GPs had contacted addictions services stating that they would not prescribe acamprosate because of local policy prohibiting its prescription from Primary Care (this policy is in fact no longer current)

Conclusion. Practice changed to offer patients monthly follow-up with addictions services for six months

Template letter sent out to GPs with discharge from addictions requesting acamprosate prescription, outlining current policy and offering support if GPs not comfortable

Audit presented to medical team. Treatment pathway amended to specify medical team's role in offering anti-craving medication at initial appointment

Re-audit in six months
Audit of high dose antipsychotic prescribing in the havering community recovery team

Chinedu Umeh* and Simona Ionita

North East London Foundation Trust

${ }^{*}$ Corresponding author.

doi: 10.1192/bjo.2021.939

Aims. The main aim of this audit was to determine the prevalence of HDAP in Havering Community Recovery Team (CRT). The secondary aim was to determine how well HDAP has been monitored and documented - specifically, whether discussions around the reasons for continuing and the risks and benefits have been discussed. Background. There is a focus to reduce high dose antipsychotic prescribing (HDAP) due to the lack of evidence that it is efficacious and that smaller doses have an equivalent effect and are better tolerated. Similarly, the consensus by the Royal College of Psychiatrists is that any prescribing of high dose antipsychotics should be an 'explicit, time-limited individual trial' with a distinct treatment target. There should be a clear plan for regular clinical review including safety monitoring. The high-dose regimen should only be continued if the trial shows evidence of benefit that is not outweighed by tolerability or safety problems.' Following a CQC inspection in 2014 of NELFT which found that the trust was failing to comply with the relevant requirements of the Health and Social Care Act 2008 with regards to safe use of medicines, yearly audits of inpatient HDAP have been undertaken. Although improvements have been made in the inpatient setting, no such audits have been performed in the community setting and consequently there is no data in NELFT regarding community services compliance with the above regulations.

Method. All 349 patients in Havering CRT clinical records were screen by either using RIO or GP letters from recent CPA reviews. A data collection and analysis tool was created using Microsoft Excel. Data collection and analysis was carried out by the project lead and checked by a fellow project member.

Result. Of the 349 patients included for analysis:

$16(4.58 \%)$ of patients were prescribed a high-dose antipsychotic

Of the 16 prescribed high dose antipsychotics:

0 out of 16 had the high dose antipsychotic monitoring form available

$12(75 \%)$ had well documented evidence of review of HDAP.

$4(25 \%)$ had no documented evidence of review of HDAP.

Conclusion. There is a small group of patients receiving high dose antipsychotic therapy for which better monitoring is needed. This should include education of staff regarding HDAP, better documentation in their care plans and working with pharmacy to make HDAP monitoring forms available widely in the community.

\section{ELPS helps!}

Rajalakshmi Valaiyapathi*, Ksenia Marjanovic-Deverill and Kezia Smith

Central North West and West London Mental Health Trust ${ }^{*}$ Corresponding author.

doi: 10.1192/bjo.2021.940

Aims. This study aimed to identify whether contact with the Ealing Liaison Psychiatry Service (ELPS) improved patients' mental health using the Clinical Global Impressions (CGI) scale, and to understand the utility of this tool. 